\title{
New host and geographical records for helminths parasites of Ardeidae (Aves, Ciconiiformes) in Brazil
}

\author{
Vanessa S. de Arruda ${ }^{1}$ \\ Roberto M. Pinto ${ }^{1,2}$ \\ Luís C. Muniz-Pereira ${ }^{1,3}$
}

\begin{abstract}
One thousand two-hundred-four helminth samples recovered from Brazilian Ardeidae birds during 54 years were studied. Samples consisted of cestodes, trematodes, and nematodes, parasites of egrets and herons. Based on the present results 49 new host and three new geographical distribution records were established.

KEY WORDS. Helminths, birds, Ciconiiformes, Ardeidae, new host and geographical distribution records, Brazil
\end{abstract}

The ardeid birds, commonly known as egrets and herons, belong to the order Ciconiiformes, generally inhabiting tropical and subtropical regions in mudflats, ponds, lakes, water reservoirs and dam areas, as well as wetlands. Ardeids feed on fishes, amphibians, reptiles, insects and mollusks, that could serve as intermediate hosts for the helminths. Some ardeids can act as definitive hosts for some potential zoonotic helminth species, such as Phagicola angrense (Travassos, 1916), playing an important role in such infections that can be transmitted to man from the same intermediate hosts.

The helminths studied herein were recovered from 12 species of ardeid birds, collected during major Brazilian parasite surveys, between 1909 and 1963, that were incorporated to the Helminthological Collection of the Oswaldo Cruz Institute (CHIOC).

\section{MATERIAL AND METHODS}

The present new records were obtained from 1204 studied samples of helminths parasites of ardeids, deposited in the Helminthological Collection of the Oswaldo Cruz Institute (CHIOC). Specimens are kept either in numbered vials or as whole mounts, matched with respective numbered files. The source of samples according to the host species is: Ardea cocoi (Linnaeus, 1766), Butorides striatus (Linnaeus, 1758), Casmerodius albus (Linnaeus, 1758), Egretta thula (Molina, 1782), Florida caerulea (Linnaeus, 1758), Ixobrichus exilis (Gmelin, 1789), Nyctanassa violacea (Linnaeus, 1758), Nycticorax nycticorax (Linnaeus, 1758), Nycti-

1) Laboratório de Hemintos Parasitos de Vertebrados, Departamento de Helmintologia, Instituto Oswaldo Cruz. Avenida Brasil 4365, 21045-900 Rio de Janeiro, Rio de Janeiro, Brasil.

2) CNPq research fellow. E-mail: rmpinto@gene.dbbm.fiocruz.br

3) Corresponding author. E-mail: Imuniz@ioc.fiocruz.br 
corax sp., Pilherodius pileatus (Boddaert, 1783), Syrigma sibilatrix (Temminck, 1824), Tigrisoma lineatum (Boddaert, 1783), representing 50\% of the Brazilian ardeid species.

Helminths were preserved in $70^{\circ} \mathrm{GL}$ ethanol, AFA, or Railliet \& Henry solution $(0,85 \mathrm{NaCl}$ solution: $93 \mathrm{ml}$; formaldehyde: $3 \mathrm{ml}$; glacial acetic acid: $2 \mathrm{ml})$ and processed for study as described elsewhere (MUNIZ-PEREIRA \& AMATO 1995, 1998; VICENTE et al. 1995a). NHR and NGR indicate New Host Record, and New Geographical Records, respectively. Classification of hosts follows SICK (1997). Determination of the digenean specimens to generic level was based on YAMAGUTI (1971) and TRAVASSOS et al. (1969). Cestodes were determined to generic level according to the keys of SCHMIDT (1986) and KHALIL et al. (1994). Assignment of the digeneans and cestodes to a specific level was obtained from the available literature. The diagnosis of the nematodes to a specific level was achieved by the catalogue of the nematodes from birds (VicENTE et al. 1995b), YAMAGUTI (1961), and SKRJABIN \& SoBOleV (1963). The classification of the major groups of Platyhelminthes follows BROOKS (1989). Voucher determined specimens were deposited in the Helminthological Collection of the Oswaldo Cruz Institute (CHIOC) as whole mounts and wet material.

\section{RESULTS \\ Platyhelminthes Gegenbauer, 1859 \\ Digenea Van Beneden, 1858}

Clinostomidae Luehe, 1901

\section{Clinostomum marginatum (Rudolphi, 1819) Braun, 1899}

NHR: Tigrisoma lineatum.

Site of infection: buccal cavity and proventriculus.

Locality: Angra dos Reis, RJ and Manguinhos, Rio de Janeiro, RJ.

Studied specimens: CHIOC 6001 (wet material); 24639 (whole mount).

Other hosts: Ardea cocoi, Butorides striatus, Casmerodius albus, Nyctanassa violacea and Nycticorax nycticorax according to TRAVASSOS (1969).

Ithyoclinostomum dimorphum (Diesing, 1850) Witenberg, 1926

NHR: Nycticorax sp. and Tigrisoma lineatum.

Site of infection: esophagus.

Locality: Pirassununga, SP (Nicticorax sp.), and São João, MS (T. lineatum).

Studied specimens: CHIOC 156, 3520, 3522, 8316 (wet material).

Other host: Ardea cocoi according to TRAVASSOS (1969).

Cyathocotylidae Poche, 1926

\section{Mesostephanus infecundus Lutz, 1935}

NHR: Butorides striatus.

Site of infection: intestine.

Locality: Rio de Janeiro, RJ.

Studied specimens: CHIOC 25449, 25450 (whole mounts). 


\section{Diplostomidae Poirier, 1886}

\section{Diplostomum sp.}

NHR: Butorides striatus and Nyctanassa violacea.

Site of infection: intestine.

Locality: Rio de Janeiro, RJ.

Studied specimens: CHIOC 24579, 24580b, 24887, 24892, 24893, 24894, 24895, 24896, 24897, 24898a, 24899b, 24900, 24901, 24902, 25349a, 25350a (whole mounts).

\section{Heterophyidae Odhner, 1914}

\section{Phagicola angrense (Travassos, 1916) Travassos, 1929}

NHR: Ardea cocoi.

Site of infection: intestine.

Locality: Manguinhos, Rio de Janeiro, RJ.

Studied specimens: CHIOC 6637 (wet material).

Other hosts: Butorides striatus, Butorides sp., Ixobrychus exilis, Nyctanassa violacea according to TRAVASSOS (1969), and YAMAGUTI (1971).

\section{Pygidiopsis pindoramensis Travassos, 1929}

NHR: Nyctanassa violacea and Nycticorax nycticorax.

Site of infection: intestine.

Locality: Manguinhos, Rio de Janeiro, RJ.

Studied specimens: CHIOC 6632, 6633 (whole mounts); 6641, 6696, 7039 (wet material).

Other host: Ixobrychus exilis according to TRAVASsOs (1969).

\section{Microphallidae Travassos, 1921}

\section{Gynaecotyla jagerskioldi (Travassos, 1920) Yamaguti, 1939}

NHR: Butorides striatus, Florida caerulea and Nyctanassa violacea.

Site of infection: intestine.

Locality: Manguinhos, Rio de Janeiro, RJ.

Studied specimens: CHIOC 2231a, 2232b, 2233, 2234, 2235, 2236, 2237, 2239a, 2589, 2590c, 2591 c, 2645, 2780, 2781a, 2782a, 2803a, 2804a, 2805a, 2806, 2897, 12040, 12095, 12096, 17515, 25361, 25368 (whole mount); 12222 (wet material).

\section{Levinseniella cruzi Travassos, 1920}

NHR: Nyctanassa violacea.

Site of infection: intestine.

Locality: Manguinhos, Rio de Janeiro, RJ.

Studied specimens: CHIOC 2238, 2239b (whole mounts); 2866, 2870, 2875 (wet material); 3080 (whole mount). 


\section{Levinseniella sp.}

NHR: Nyctanassa violacea and Nycticorax nycticorax.

Site of infection: intestine.

Locality: Manguinhos, Rio de Janeiro, RJ.

Studied specimens: CHIOC 2664, 2665, 2666, 2667, 2694, 17443, 17496, 25349, 25350, 25352, 25353, 25361b (whole mounts); 2865, 2894, 5991, 7165, 8348 (wet material).

\section{Maritrema nicolli Travassos, 1920}

NHR: Florida caerulea and Nyctanassa violacea.

Site of infection: intestine.

Locality: Manguinhos, Rio de Janeiro, RJ.

Studied specimens: CHIOC 2585, 2586, 2587, 2588, 2590a, 2591a, 2646, 2803b, 2804b, 2805b (whole mounts).

\section{Maritrema sp.}

NHR: Butorides striatus and Nyctanassa violacea.

Site of infection: intestine. violacea).

Locality: Brazil (B. striatus), and Manguinhos, Rio de Janeiro, RJ (N.

Studied specimens: CHIOC 18056 (wet material); 25368 (whole mount).

\section{Opisthorchiidae Braun, 1901}

\section{Amphimerus interruptus (Braun, 1909) Barker, 1911}

NHR: Ardea cocoi, Nycticorax nycticorax, Pilherodius pileatus, Syrigma sibilatrix and Tigrisoma lineatum.

Site of infection: pancreas and gall bladder.

Locality: São João, MS.

Studied specimens: CHIOC 3488 (whole mount); 3497, 3510, 3511, 3512, 3513 (wet material).

Other host: Butorides striatus ? according to TRAVASSOS (1969).

\section{Philophtalmidae Travassos, 1918}

\section{Philophtalmus lacrymosus Braun, 1902}

NHR: Nyctanassa violacea.

Site of infection: eye.

Locality: Rio de Janeiro, RJ.

Studied specimens: CHIOC 861 (whole mount); 17964 (wet material).

Other host: Casmerodius albus according to TRAVASSOS (1969).

Proctobium proctobium Travassos, 1918 (Travassos, 1921)

NHR: Ardea cocoi and Egretta thula.

Site of infection: Bursa Fabricii, cloaca, and intestine. 
Locality: São João, MS (A. cocoi), and Manguinhos, Rio de Janeiro, RJ ( $E$. thula).

Studied specimens: CHIOC 33978, 34241 (whole mounts).

Other host: Nyctanassa violacea according to TRAVASSOS (1969).

Stomylotrematidae Poche, 1926

Stomylotrema gratiosus Travassos, 1922

NHR: Syrigma sibilatrix.

Site of infection: cloaca.

Locality: Salobra, MS.

Studied specimens: CHIOC 11278 (whole mount).

Strigeidae Railliet, 1919

\section{Apatemon globiceps Dubois, 1937}

NHR: Nyctanassa violacea.

Site of infection: intestine.

Locality: Brazil.

Studied specimens: CHIOC 24548 (whole mount).

Apharyngostrigea brasiliana (Szidat, 1928) Szidat, 1929

NHR: Butorides striatus and Nyctanassa violacea.

Site of infection: intestine. violacea).

Locality: Brazil (B. striatus), and Manguinhos, Rio de Janeiro, RJ ( $N$.

Studied specimens: CHIOC 17203, 17204, 17218, 24551 (whole mounts).

\section{Eucestoda}

\section{Dilepididae Railliet \& Henry, 1909}

\section{Anomotaenia sp.}

NHR: Ardea cocoi.

Site of infection: intestine.

Locality: Ilha de Marajó, PA.

Studied specimens: CHIOC 10142 (wet material).

\section{Dendrouterina sp.}

NHR: Ardea cocoi.

Site of infection: intestine.

Locality: Salobra, MS.

Studied specimens: CHIOC 34031(whole mount).

\section{Dendrouterina pilherodiae Mahon, 1956}

NHR: Casmerodius albus.

Site of infection: intestine. 
Locality: Gaspar Lopes, Alfenas, MG.

Studied specimens: CHIOC 30728 a-d (whole mounts).

Other host: Pilherodius pileatus according to SCHMIDT (1986).

\section{Dilepis fuhrmanni Railliet \& Henry, 1909}

NHR: Egretta thula.

Site of infection: intestine.

Locality: Manguinhos, Rio de Janeiro, RJ.

Studied specimens: CHIOC 1022 (whole mount); 1023, 2388 (wet material).

\section{Valipora sp.}

NHR: Pilherodius pileatus.

Site of infection: intestine.

Locality: Urucum, MS.

Studied specimens: CHIOC 20578, 20579 (wet material); 34077, 34078 (whole mounts).

\section{Hymenolepididae Ariola, 1849}

\section{Diploposthe bifaria Siebold, 1946}

NHR: Butorides striatus.

NGR: Salobra, MS.

Site of infection: intestine.

Studied specimens: CHIOC 34043 (whole mount).

This species was referred to Europe, Asia, Africa, Australia and North America according to KHALIL et al. (1994).

\section{Jardugia sp.}

NGR: Exu, PE.

Host: Ardeidae sp.

Site of infection: intestine.

Studied specimens: CHIOC 34081a-b (whole mounts).

Species from this genus was referred in Nigeria by KHALIL et al. (1994).

\section{Drepanidotaenia sp.}

NHR: Ardea cocoi, Butorides striatus, Butorides sp., Ixobrychus exilis.

Site of infection: intestine.

Locality: Salobra, MS, (A. cocoi, and B. striatus), Bodoquena, MS (B. striatus), Angra dos Reis, RJ (Butorides. sp., and B. striatus) and Manguinhos, Rio de Janeiro, RJ (I. exilis).

Studied specimens: CHIOC 34032, 34042, 34045, 34090, 34093, 34094, 34104 (whole mounts). 


\section{Nematoda Rudolphi, 1808 \\ Spiruroidea Railliet \& Henry, 1915}

\section{Spiruroidea sp.}

NHR: Casmerodius albus.

Site of infection: esophagus.

Locality: Salobra, MS.

Studied specimens: CHIOC 12777 (wet material).

\section{Acuariidae Seurat, 1913}

\section{Acuaria sp.}

NHR: Nyctanassa violacea.

Site of infection: proventriculus.

Locality: Manguinhos, Rio de Janeiro, RJ.

Studied specimens: CHIOC 494, 1809, 1810, 2830, 2832 (wet material).

\section{Cheilospirura hamulosa (Diesing, 1851) Diesing, 1861}

NHR: Nyctanassa violacea and Syrigma sibilatrix.

Site of infection: proventriculus and peritoneum. sibilatrix).

Locality: Rio de Janeiro, RJ (N. violacea), and Barranco Alto, MS ( $S$.

Studied specimens: CHIOC 14198 (wet material); 34238 (whole mount).

\section{Dracunculidae Leiper, 1912}

\section{Avioserpens sp.}

NHR: Nycticorax nycticorax.

NGR: Manguinhos, Rio de Janeiro, RJ.

Site of infection: esophagus.

Studied specimens: CHIOC 9156 (whole mount).

Species from genus Avioserpens were referred in North America, France, India and Asia (Yamaguti 1961). This is the first geographical record for South America.

\section{Onchocercidae (Leiper, 1911)}

\section{Pelecitus sp.}

NHR: Syrigma sibilatrix and Tigrisoma lineatum.

Site of infection: among tendons of feet.

Locality: Barranco Alto, MS.

Studied specimens: CHIOC 14204, 14206, 14479, 14602 (wet material); 34023 (whole mount). 


\title{
Tetrameridae Travassos, 1914
}

\section{Tetrameres cochleare Travassos, 1917}

NHR: Nycticorax nycticorax.

Site of infection: proventriculus.

Locality: Manguinhos, Rio de Janeiro, RJ.

Studied specimens: CHIOC 3553 (whole mount).

\begin{abstract}
ACKNOWLEDGEMENTS. To Dr Dante M Teixeira, head of the Ornithological Section, Museu Nacional do Rio de Janeiro (UFRJ) and to Prof. Jorge Bruno Nacinovic, Ornithological Section Museu Nacional do Rio de Janeiro (UFRJ), for reviewing the host nomenclature and discussing about host taxonomical status; to Prof. Joaquim Júlio Vicente, FIOCRUZ Department of Helminthology, for helping in some nematode species determination; to Prof. Dely Noronha, FIOCRUZ Department of Helminthology (CHIOC), for providing the deposited helminth specimens.
\end{abstract}

\section{REFERENCES}

BRooKs, D.R. 1989. A summary of the data base pertaining to the phylogeny of the major groups of parasitic Platyhelminthes, with a revised classification. Can. Jour. Zool. 67: 714-720.

Khalil, L.F.; A. Jones \& R.A. BraY. 1994. Key to the cestode parasites of vertebrates. CAB International, University Press, Cambridge, $751 \mathrm{p}$.

Muniz-PereirA, L.C. \& S.B. Amato. 1995. Natural hosts of Notocotylus breviserialis (Digenea, Notocotylidae) parasite of Brazilian waterfowl. Mem. Inst. Oswaldo Cruz 90: 711-714.

Muniz-Pereira, L.C. \& S.B. Amato. 1998. Fimbriaria fasciolaris and Cloacotaenia megalops (Eucestoda, Hymenolepididae), cestodes from Brazilian waterfowl. Mem. Inst. Oswaldo Cruz. 93: 767-772.

SCHMIDT, G.D. 1986. Handbook of tapeworm identification. Boca Raton, Florida, C.R.C. Press. Inc., $675 \mathrm{p}$.

Sick, H. 1997. Ornitologia Brasileira. Rio de Janeiro, Nova Fronteira, 827p.

SkRJABin, K.I. \& A.A. Sobolev. 1963. Treatise of Nematodology. Spirurata from animals and men and diseases caused by them. Moscow, Akad. Nauk SSSR Ed., Vol. 11, Russian text, 511p.

Travassos, L.; J.F.T. Freitas \& A. Kohn. 1969. Trematódeos do Brasil. Mem. Inst. Oswaldo Cruz 67: 1-886.

Vicente, J.J.; R.M. Pinto; D. Noronha \& L. GonçAlves. 1995a. Nematode parasites of Brazilian Ciconiiformes birds: a general survey with new records for the species. Mem. Inst. Oswaldo Cruz 90 (3): 389-393.

Vicente, J.J.; H.O. Rodrigues; D.C. Gomes \& R.M. Pinto. 1995b. Nematóides do Brasil. Parte IV: nematóides de aves. Revta bras. Zool. 12 (Supl. 1): 1-273.

YAMAGUTI, S. 1961. Systema helminthum. The nematodes of vertebrates. New York, Interscience Publishers, 679p.

- 1971. Synopsis of digenetic trematodes of vertebrates. Tokyo, Keigaku Publ., 1074p.

Recebido em 19.IX.2000; aceito em 26.VI.2001.

Revta bras. Zool. 18 (Supl. 1): 225 - 232, 2001 